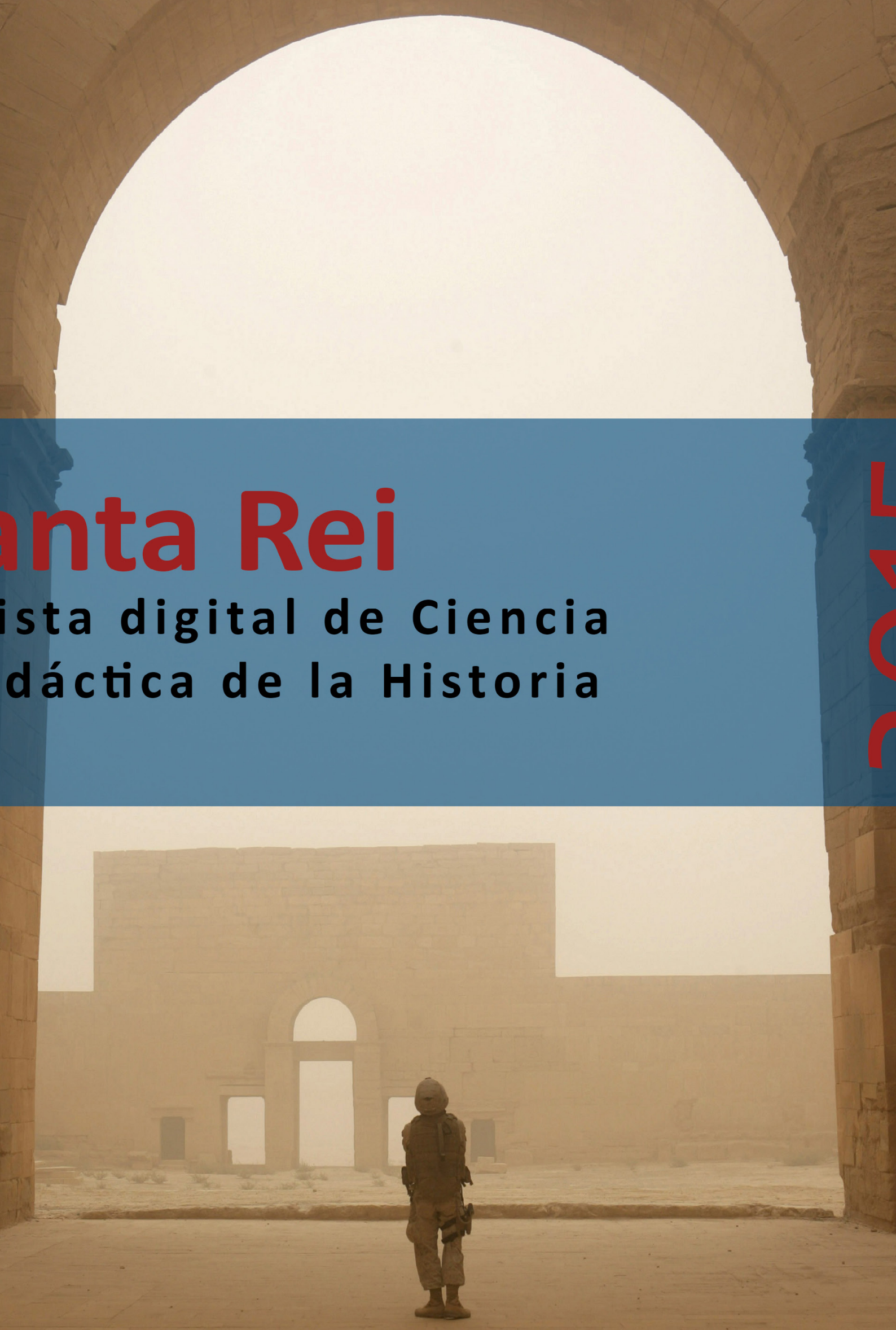

Revista digital de Ciencia y Didáctica de la Historia 


\section{Panta Rei \\ Revista Digital de Ciencia \\ y Didáctica de la Historia}

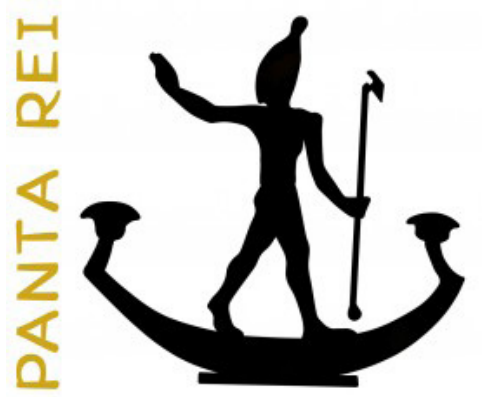

\section{5}

Revista anual

Fecha de inicio: 1995

Revista Panta Rei. pantarei@um.es

Edita:

Centro de Estudios del Próximo Oriente y la Antigüedad Tardía - CEPOAT

Edificio Universitario Saavedra Fajardo.

Universidad de Murcia

C/ Actor Isidoro Máiquez, 9

30007 - MURCIA - ESPAÑA

Teléfono: (+34) 868883890

cepoat@um.es

Web: www.um.es/cepoat/pantarei

Edición 2015

ISSNe: 2386-8864

ISSN: 1136-2464

Depósito legal: MU-966-1995
cepoAt

UNIVERSIDAD DE MURCIA centro de estudios del próximo oriente y la antigüedad tardía

En Portada: Ruinas de Hatra (Irak) (Fotografía de: Lance Cpl. Albert F. Hunt, U. S. Marine Corps).

Responsables de los textos: Sus autores.

Responsable de la presente edición: Consejo Editorial Panta Rei. 


\section{CONSEJO DE REDACCIÓN}

\section{Coordinador editorial}

Egea Vivancos, Alejandro

[Didáctica de las Ciencias Sociales, UMU]

\section{Editores}

Botí Hernández, Juan Jesús

[CEPOAT, UMU]

Meseguer Gil, Antonio José

[CEPOAT, UMU]

Sáez Giménez, David Omar

[CEPOAT, UMU]

Sánchez Mondéjar, Celso Miguel

[CEPOAT, UMU]

\section{Secretaria}

Arias Ferrer, Laura

[Didáctica de las Ciencias Sociales, UMU]

\section{Responsable informático}

Martínez García, José Javier

[CEPOAT, UMU]

\section{Traducción y corrección lingüística}

Martínez Martínez, Cristina

[Sociedad Española de Lenguas Modernas]

\section{CONSEJO ASESOR}

Albero Muñoz, M. ${ }^{a}$ del Mar [H. ${ }^{a}$ del Arte, UMU]

Chapman, Arthur

[History Education, UCL, Reino Unido]

Cobacho López, Ángel

[Derecho, UMU]

Egea Bruno, Pedro M. ${ }^{\text {a }}$

[Historia Contemporánea, UMU]

García Atienzar, Gabriel

[Prehistoria, UA]

González Monfort, Neus

[Didáctica de las Ciencias Sociales, UAB]

Haber Uriarte, María

[Prehistoria, UMU]

Irigoyen López, Antonio

[Historia Moderna, UMU]

Mahony, Simon

[Digital Humanities, UCL, Reino Unido]

Marsilla de Pascual, Francisco Reyes

[Técnicas historiográficas, UMU]

Miralles Maldonado, José Carlos

[Filología Clásica, UMU]

Molina Gómez, José Antonio

[Historia Antigua, UMU]

Noguera Celdrán, José Miguel

[Arqueología, UMU]

Pérez Molina, Miguel Emilio

[Filología Clásica, UMU]

Prados Martínez, Fernando

[Arqueología, UA]

Sánchez Ibáñez, Raquel

[Didáctica de las Ciencias Sociales, UMU]

Sancho Gómez, Miguel Pablo

[Educación, UCAM]

Vilar García, María José

[Historia Contemporánea, UMU] 

Artículos

El patrimonio arqueológico iraquí y su destrucción a lo largo del tiempo.

Fernando Espejel Arroyo.

Tacita Muta y el silencio femenino como arma del patriarcado romano.

Sara Casamayor Mancisidor.

Espartaco en la arqueología.

Juan Luis Posadas.

La batalla de Andagoste.

Luis Amela Valverde.

Las trabas para una formación arqueológica inclusiva.

Ana Samaniego Espinosa.

Repensando las narrativas nacionales: Un análisis del origen, transmisión e influencia en el aprendizaje histórico.

César López Rodríguez

El profesor como predicador, provocador y ermitaño. Un pre-texto para pensar las tareas de la didáctica de la historia.

Enrique Ibáñez Rojo

Entrevista

Evaluando el desarrollo de la competencia histórica en la enseñanza de la Historia en España. Entrevista a Jesús Domínguez Castillo.

María José Morales Rodríguez.

Reseñas

II Congreso de Jóvenes Investigadores del Mundo Antiguo de la Universidad de Murcia.

Pedro David Conesa Navarro y Juana M. ${ }^{a}$ Martínez Molina.

II Jornadas Internacionales de Didáctica de la Historia.

Eusebio Manuel Vidal Abellán.

Dölger, F. J. (2015). La luz de Cristo. Madrid: Ediciones Encuentro. 98 págs.

Alejandro Ruiz Sánchez.

Cooper, H. (ed.) (2013). Teaching History Creatively. Londres: Routledge. 185 págs.

Marta Gil de Sola Bellas.

El arte paleolítico en El Carnicero de Chabrol.

Alberto Lombo Montañés.

Normas de publicación/Publishing rules 



\title{
La batalla de Andagoste
}

\author{
The Battle of Andagoste
}

\author{
Luis Amela Valverde ${ }^{1}$ \\ Grupo CEIPAC. Universidad de Barcelona
}

Recibido: 06/03/15

Aceptado: 18/08/15

Para citar este artículo: Amela Valverde, L. (2015). La batalla de Andagoste. Panta Rei. Revista Digital de Ciencia y Didáctica de la Historia, 51-61.

ISSNe: 2386-8864

DOI: http://dx.doi.org/10.6018/pantarei/2015/4

\section{Resumen}

Si bien las Guerras Cántabras (29-19 a.C.) se inician tras consolidar Octaviano/Augusto su poder personal en Roma, la celebración de una serie de triunfos ex Hispania por parte de los últimos gobernadores republicanos peninsulares parece indicar que las operaciones militares contra los últimos grupos étnicos peninsulares independientes se iniciaron mucho antes. El hallazgo de un posible campamento y/o campo de batalla en Andagoste es la verificación arqueológica de este aserto.

\section{Palabras clave}

Historia Antigua, Arqueología, Hispania, Sitio arqueológico.

\section{Abstract}

While the Cantabrian Wars (29-19 BC) were initiated after the consolidation of the personal power of Octavian/Augustus in Rome, the celebration of a series of triumphs ex Hispania by the last peninsular Republican governors, suggests that military operations against the last independent peninsular ethnic groups began much earlier. The finding of a possible camp and/or battlefield in Andagoste is the archaeological verification of this assertion.

\section{Keywords}

Ancient History, Archaeology, Hispania, Archaeological site.

\footnotetext{
1 Para contactar con el autor: Luis Amela Valverde. Investigador Colaborador. Centro para el estudio de la interdependencia provincial en la Antigüedad Clásica. Departament de Prehistòria, Història Antiga y Arqueologia. Facultat de Geografia i Història, Universitat de Barcelona. C/ Montalegre 6, 08001 Barcelona, España. amelavalverde@gmail.com
} 


\section{Introducción}

El lugar de Andagoste (Cuartango, Álava), ubicado en la parte occidental de la Comunidad Autónoma del País Vasco, es una suave elevación de terreno $(624 \mathrm{~m})$ situado en el centro del valle del Cuartango, sobre la confluencia del río Bayas con su pequeño afluente el Vadillo, que corre desde el oeste. Andagoste es uno de los escalones más bajos de la Sierra de Guillarte, divisoria de aguas de la cuenca del Nervión y la cuenca del Bayas, afluente del río Ebro. El emplazamiento se encuentra rodeado por las altas alineaciones montañosas que configuran Cuartango, cerrando el valle por el sur y el oeste (Sierra de Arcamo, $1184 \mathrm{~m}$ ), por el este (Sierra de Bedaya, $1042 \mathrm{~m}$ ) y la citada Sierra de Guillarte por el norte (Ocharán y Unzueta, 2002, 2005; Unzueta, 2007; Unzueta y Ocharán, 1999, 2006).

Los accesos a Andagoste son extremadamente accidentados: por su lado meridional, el valle de Cuartango sólo está abierto a través de la garganta de Techa o Subijana, sellada profundamente en las peñas calizas por el río Bayas, mientras que las salidas oriental y septentrional deben salvar los altos de Izarra y Altube, en la divisoria de aguas entre las vertientes atlántica y mediterránea. De esta forma, Cuartango es un punto estratégico para el control de paso norte-sur entre la franja costera cantábrica vizcaína y la cuenca del Ebro (Morillo, 2003; Ocharán y Unzueta, 2002; Unzueta y Ocharán, 1999, 2006).

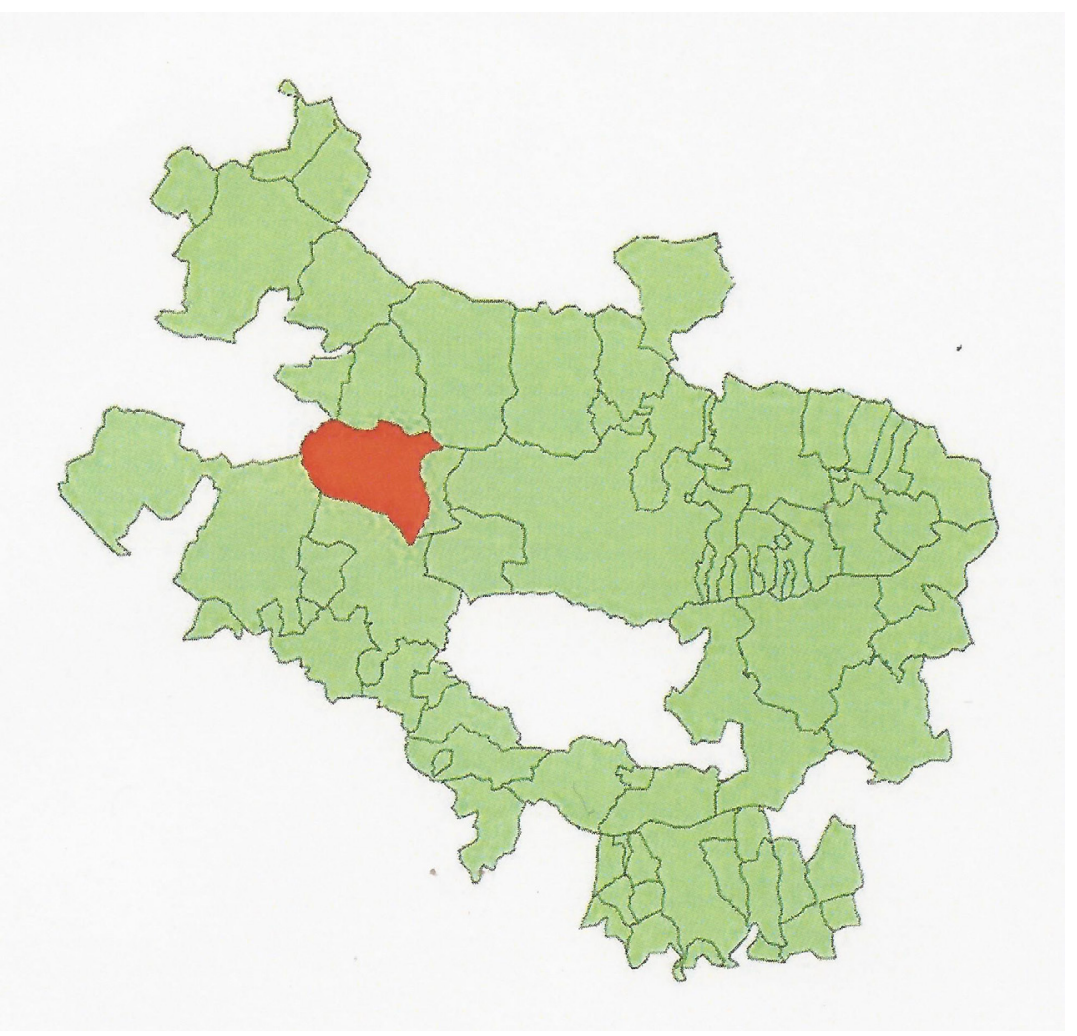

Figura 1: Ubicación del municipio de Cuartango en la provincia de Álava. Fuente: https://es.wikipedia.org/wiki/Cuartango 


\section{Descubrimiento}

En la colina de Andagoste, durante las obras de colocación de una nueva red de abastecimiento de agua potable para el municipio de Cuartango, se recogió en superficie diversos materiales de filiación militar, cuya excavación ha permitido localizar un posible campamento y/o campo de batalla de cronología tardorrepublicana (Gil, 2002; Ocharán y Unzueta, 2002), en el que se han hallado monedas, elementos de sujeción para correajes, objetos de ornato o vestimenta, proyectiles (lo que implica la presencia de una catapulta para lanzarlos), armamento ligero de todo tipo (Gil, 2002; Morillo, 2003; Ocharán y Unzueta, 2002; Unzueta y Ocharán, 1999, 2006), que contrasta con la total ausencia de materiales cerámicos y restos estructurales de fábrica en piedra o adobe, lo que lleva a suponer la inexistencia de un yacimiento estable de larga duración (Amela, 2006, 2009; Ocharán y Unzueta, 2002; Unzueta y Ocharán, 1999, 2006).

Por tanto, no existe evidencia alguna para justificar la presencia en Andagoste de un asentamiento indígena que pudiera haber sido atacado por fuerzas militares romanas, que originaría la presencia de los diferentes tipos de proyectiles hallados en el yacimiento. Asimismo, tampoco puede defenderse la existencia en este lugar de un asentamiento romano imperial que, en algún momento de su desarrollo, hubiera albergado un contingente militar que justificara los materiales recuperados citados anteriormente. La carencia de evidencias confirma pues la ocupación temporal del lugar, en un momento cronológico concreto y de manera puntual (Ocharán y Unzueta, 2002; Unzueta y Ocharán, 1999, 2006).

\section{Un campo de batalla}

Como podremos observar a continuación, parece ser que en Andagoste se ha localizado un campo de batalla fortificado a toda prisa por parte de una unidad militar romana (Morillo, 2003) o una posición defensiva romana (Unzueta y Ocharán, 1999), que sería atacado por parte de fuerzas indígenas ${ }^{2}$. Por la distribución de pueblos admitida generalmente, se tratarían de integrantes de la etnia de los autrigones, aunque no se puede descartar que participaran miembros de otros grupos vecinos, como los caristios y los várdulos.

Se ha considerado que el contingente militar romano estaría formado por una legión "miliaria" (sic) o de tres a cuatro cohortes (Fernández, 2002), aunque no se especifica el porqué de este número de unidades. El cálculo ha de basarse en la extensión del campamento, por lo que podría haber albergado a dos o tres cohortes (1200 a 1800 hombres de manera teórica), más auxiliares (Ocharán, 2006), aunque es difícil conocerlo de manera exacta debido a que no se ha excavado el yacimiento en su totalidad.

Asimismo, algunos consideran que este campo de batalla presenta grandes semejanzas de tipo formal con el descubierto en fecha reciente en la actual ciudad de Valencia, en la cual se abrieron varios fosos para la defensa de la población. Valentia fue tomada de manera violenta en el año 75 a.C. por las tropas de Cn. Pompeyo Magno (cos. I 70 a.C.), en el marco de la Guerra Sertoriana (83-72 a.C.) (Morillo, 2003).

2 Sobre la batalla de Andagoste existe un centro de interpretación ubicado en la Casa Troncal de Urbina de Basabe, en el municipio de Cuartango, junto a una exposición etnográfica sobre las costumbres y usos tradicionales de este valle. Este centro fue inaugurado el día 28 de marzo del año 2009. 


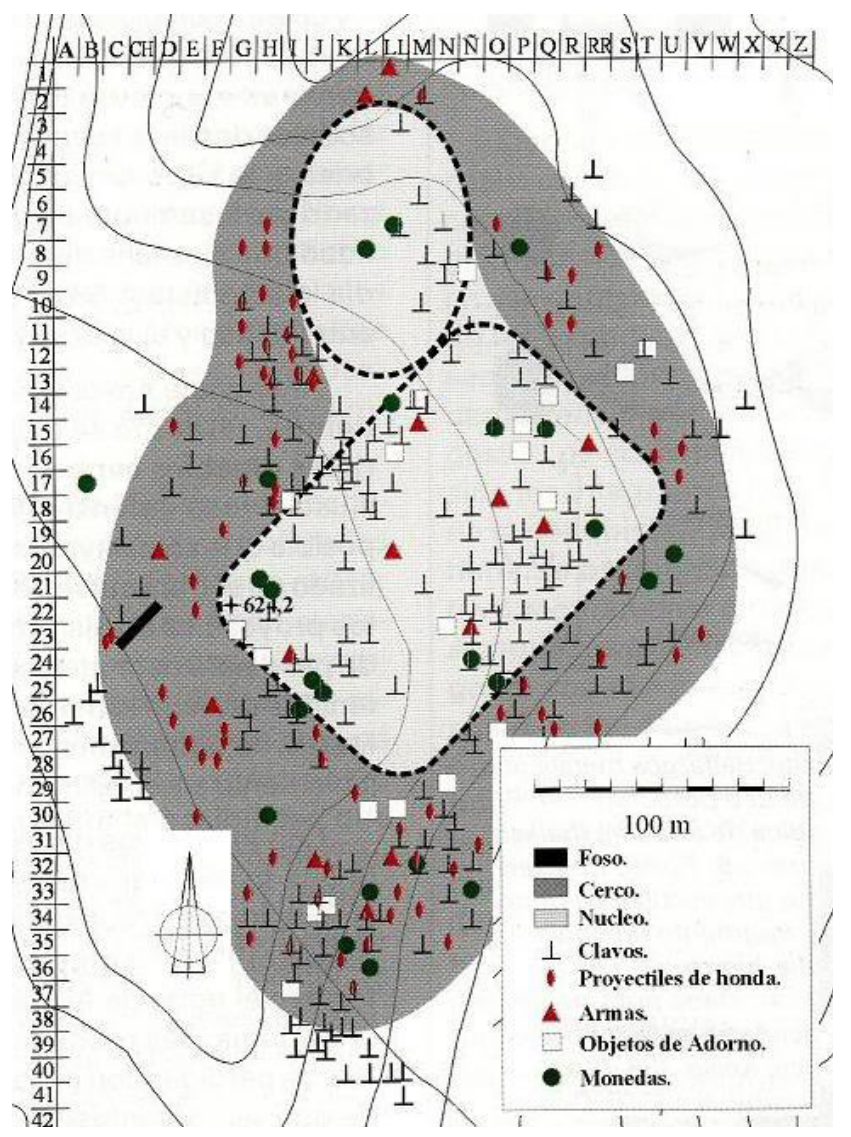

Figura 2: Plano de la excavación del yacimiento de Andagoste (según Unzueta; Amela, 2014, p. 27).

\section{Campañas de Excavación}

Se ha procedido a efectuar en este lugar un total de cinco campañas (1998-2002) de prospección geofísica (magnética y electromagnética), acompañadas de catas (Ocharán y Unzueta, 2005, 2006; Unzueta, 2007). En función de la dispersión de los materiales detectados, se pudo establecer un área fértil de 6,6 ha (es decir, $66.000 \mathrm{~m}^{2}$ ) sobre la colina de Andagoste, con especial incidencia sobre su parte alta y las laderas norte, este y sur, las cuales a su vez se subdividieron en otras dos áreas menores, "núcleo" y "cerco" respectivamente, en función de la diversidad de los materiales recuperados en ellas (Ocharán y Unzueta, 2002, 2005; Unzueta, 2007; Unzueta y Ocharán, 1999, 2006) (vid. infra).

Los indicios estructurales localizados hasta el momento son escasos, y no permiten discernir si nos encontramos ante un campamento ya sea de marcha o temporal (castra stativa) o de un simple sistema de trincheras y fosos propios de un campo de batalla (Ocharán y Unzueta, 2002). EI rasgo más notable es un simple foso de fondo plano tallado en la roca caliza que conforma la base de la colina, de un ancho de $2.40 \mathrm{~m}$ (aproximadamente 8 pies) y $0.60 \mathrm{~m}$ de profundidad (2 pies), de fondo plano, con los lados en ángulo de $45^{\circ}$, con más de $20 \mathrm{~m}$ de largo (Ocharán y Unzueta, 2002). La amplitud del foso y la inclinación de las paredes cumplen con el módulo habitual en este tipo de defensa pero no ha sido rematado en el característico perfil en $\mathrm{V}$, por lo cual pudiera pensarse que este último sería más bien un modelo, menos frecuente o, quizás, se esté frente a una obra 
inconclusa (Ocharán y Unzueta, 2002, 2005; Unzueta y Ocharán, 1999, 2006).

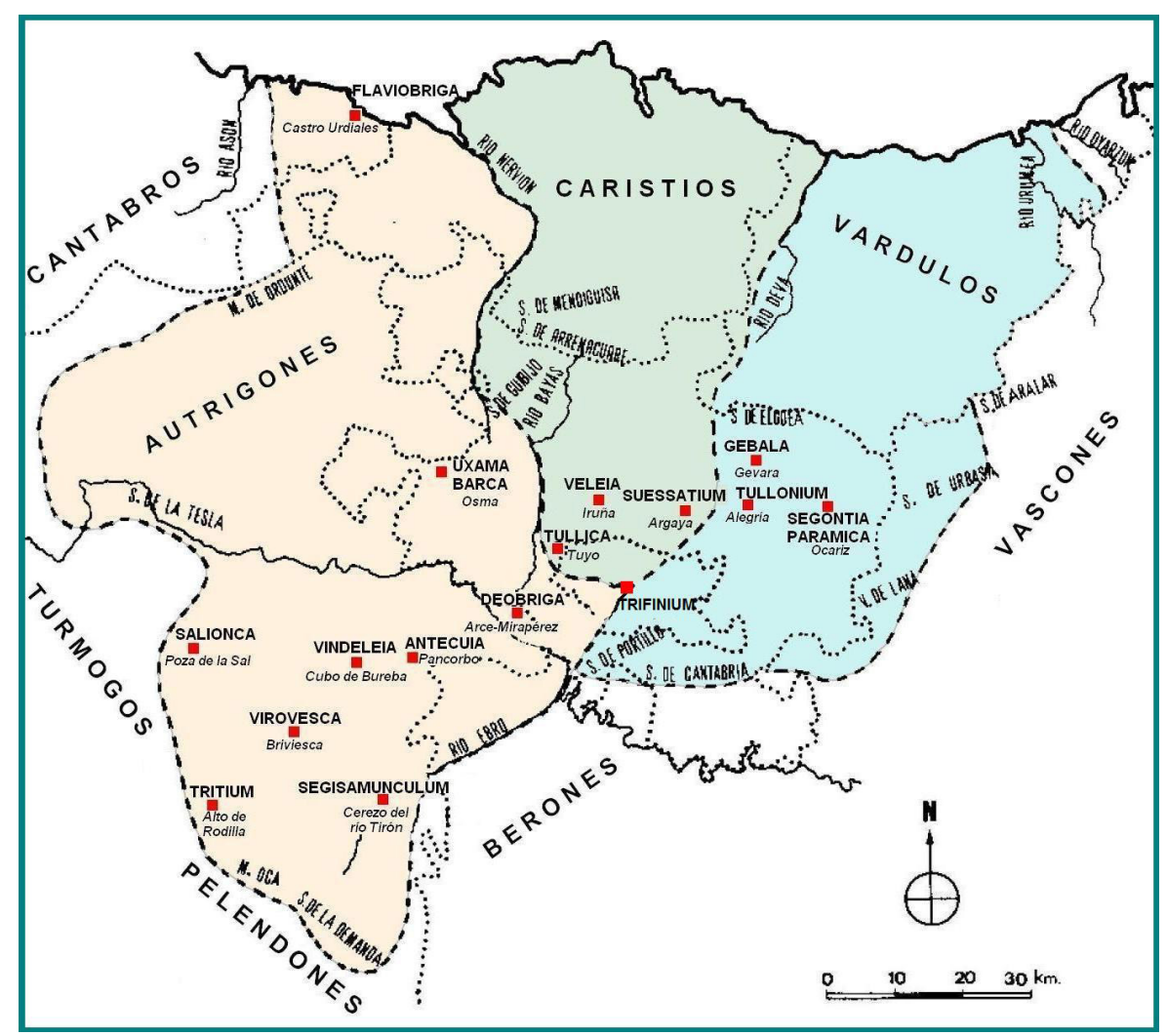

Figura 3: Límites geográficos entre Várdulos, Caristios y Autrigones según Santos, Emborujo y Ortiz de Urbina (1992, p. 456). Fuente: https://es.wikipedia.org/wiki/Autrigones

La colina contiene una escasa capa de tierra, cuya potencia oscila entre 20 y $30 \mathrm{~cm}$, roturada en esta zona. Esto ha causado la colmatación del foso y el arrasamiento de cualquier tipo de terraplén que pudiera ser considerado como un posible agger (Ocharán y Unzueta, 2002; Unzueta y Ocharán, 1999). Asimismo, se han localizado dos largos y paralelos terraplenes de tierra en las laderas norte y este, realzados por su parte delantera mediante una ancha excavación que han dejado en este punto la roca base a la vista; estas estructuras rodean en paralelos el contorno de la colina por las laderas norte y este, limitando por estos puntos la del área fértil y quizás aquí pudiera estar uno de los indicios más útiles, situándose entre ambas el sector que por estos lados más proyectiles de honda ha ofrecido (Amela, 2006; Morillo, 2003, 2009; Ocharán y Unzueta, 2002; Unzueta, 2007; Unzueta y Ocharán, 1999, 2006).

\section{Objetos arqueológicos descubiertos}

No sólo hay que dar importancia a la estructura defensiva descubierta sino también a los restos arqueológicos encontrados. Llama poderosamente la atención la presencia de un alto número de elementos de sujeción para correajes, de clara raigambre militar, algunos tipos varias veces repetidos, en forma de hebillas, tensores, pasadores, fíbulas, etc. A destacar una fíbula tipo Alesia, que corresponde al tipo 19.1.a de la tipología establecida por R. Erice, fechada entre mediados del s. I a.C. y los inicios del periodo augusteo (Ocharán y Unzueta, 2002, 2005; Unzueta, 2007; Unzueta y Ocharán, 1999, 2006).

También se ha de destacar entre el conjunto de evidencias, debido a su alta frecuencia (681 piezas, el $68 \%$ del total de elementos arqueológicos localizados en el yacimiento de Andagoste) y su amplia dispersión, un tipo de remache de hierro, conocido como "tachuela de cabeza hemisférica", 
rebajado y con un pequeño vástago de sección cuadrada, cuyo extremo, en caso de conservarse, se encuentra doblado formando un ángulo recto (Ocharán y Unzueta, 2002, 2005; Unzueta, 2007; Unzueta y Ocharán, 1999, 2006). Este tipo de tachuela presenta en su cara inferior, con ligeras variaciones, un motivo trabajado en relieve el cual, partiendo del vástago central traza, mediante cuatro radios, cuatro cuartos de círculo. Dentro de cada una de estas cartelas, y en el centro, ha sido dispuesta una pequeña semiesfera en relieve. Tanto por la forma de la cabeza como por la característica doblez del vástago parecen indicar que se tratan de remaches para guarniciones de cuero, fuese de sandalia, de escudos o de correajes (Ocharán y Unzueta, 2002, 2005; Unzueta y Ocharán, 1999, 2006).

La presencia de un número tan elevado de estos refuerzos, posiblemente de calzado (caligae), únicamente queda justificada por una utilización generalizada por los ocupantes de este asentamiento. Ante todo, el principal dato que ha suministrado las tachuelas es el de su amplia dispersión. Precisamente, en función de esta distribución, los arqueólogos responsables de la excavación han podido establecer la localización del yacimiento sobre la zona alta de la colina de Andagoste, y en sus laderas norte, este y sur, ocupando una superficie total de 6,6 ha, como ya se ha indicado.

Igualmente, junto al material citado, se recuperó un lote de armas ofensivas, sobre todo proyectiles. De esta forma, ha de señalarse la presencia de varias armaduras de dardo para balistas en hierro, entre las que se encuentran cinco de forma piramidal y sección cuadrada, rematadas en su base por una pequeña espiga cuya finalidad pudiera ser su encastramiento en el mástil del proyectil. También se localizó otro modelo de dardo de enmangue tubular, cuyo volumen y peso, al igual que los anteriores, era necesario la utilización de una máquina de artillería (de torsión) para su lanzamiento. Ello apunta a la presencia de una unidad legionaria en el lugar. Por último, también resulta significativa la presencia de una punta de pilum al igual que la de una flecha, lo que redunda en el carácter militar del conjunto (Gil, 2002; Ocharán y Unzueta, 2002, 2005; Unzueta y Ocharán, 1999, 2006).

Pero, sin embargo, el lote más llamativo y útil, debido tanto a su abundancia como por la información que puede extrapolarse de su dispersión, lo forma el conjunto de proyectiles de honda fundidos en plomo de las que se han encontrado un total de 111 piezas, el $11 \%$ del total. Son piezas, siempre anepigráficas, de forma homogénea que, con ligeras variantes, se aproximan a los perfiles bicónicos y losángicos (Ocharán y Unzueta, 2002, 2005; Unzueta, 2007; Unzueta y Ocharán, 1999, 2006).

La distribución de las glandes permite reconstruir la historia del yacimiento, al menos, en sus momentos finales. Parece ser significativo la distribución de estos proyectiles sobre la zona periférica del área fértil del yacimiento, previamente definida por la dispersión de los remaches de cabeza esférica; a excluir el espacio central, en donde no se recuperó ninguna bala de honda (Ocharán y Unzueta, 2002; Unzueta y Ocharán, 1999, 2006).

De esta forma, el espacio en el que se concentran los proyectiles queda conformado como una franja de aproximadamente 60 metros de ancho que rodea la colina de Andagoste en su totalidad. Esta área, que los excavadores del yacimiento han denominado como "cerco", es en donde ha de situarse el campo de batalla o frente de combate (Ocharán y Unzueta, 2002, 2005; Unzueta y Ocharán, 1999, 2006). Por el contrario, debido precisamente a la total ausencia de glandes, puede definirse otra zona, envuelta por la anterior área, que consta de una superficie interna de aproximadamente 2,3 ha, a la que se le ha denominado "núcleo"; ésta puede descomponerse en dos figuras geométricas: una extensa, de forma rectangular, de 17.600 metros $^{2}(160 \times 110)$, y otra menor, oval, de 5.122 metros $^{2}(110 \times 70)$, que parece corresponder a la ubicación de la posición militar romana (Ocharán y Unzueta, 2002, 2005; Unzueta y Ocharán, 1999, 2006). 


\section{Diferenciación de ámbitos con objeto de estructurar el combate}

La articulación en dos posibles ámbitos que origina la peculiar morfología de la zona denominada como "núcleo" ha de explicarse por la distribución en la que se dispusieron las tropas para la defensa, en el caso de ser sólo una posición de batalla. Sea como fuere, para los arqueólogos responsables de la excavación, sería más factible ver el área como un solar ocupado por un asentamiento campamental compuesto por dos recintos contiguos; esta división quizás fuera debida a la necesidad de acuartelar a dos unidades de diferentes armas o procedencias, como pudiera ser la combinación de un cuerpo legionario (que ocuparía la zona rectangular) y una unidad de caballería o de auxiliares (que ocuparía el área oval) (Ocharán y Unzueta, 2002; Unzueta y Ocharán, 1999, 2006).

La anterior hipótesis se basa en la distribución del material recuperado, en especial y, sobre todo, en la clara dispersión periférica de los proyectiles de honda. Ha de suponerse que estas balas de plomo, munición de uso militar, fueron arrojadas por los defensores del "núcleo", cayendo sobre la zona externa de las defensas que forman el "cerco", sobre los lugares donde se ubican el foso y los terraplenes ya documentados (Ocharán y Unzueta, 2002; Unzueta y Ocharán, 1999, 2006).

En relación a lo acontecido en este lugar, hay que señalar la no coincidencia de la estructura excavada en forma de foso con el perímetro de la zona del núcleo, quedando los fosos englobados en el cerco por su lado sur-oeste, es decir, en el lugar donde no se llegó a concluir el recinto fortificado. De esto parece deducirse que el primer intento de fortificación fue superado por el atacante, obligando a la fuerza militar romana a defenderse sobre la ladera oriental de la colina de Andagoste, perdiendo así el beneficio que le proporcionaba su posición en la zona alta de la misma (Unzueta y Ocharán, 2006).

Los responsables de la excavación señalan las reducidas dimensiones del núcleo, que en su sector más amplio sólo presenta 154 metros de ancho por 202 metros de largo, que estaría, de forma evidente, en función de los defensores. Por ello, debido a las dimensiones propuestas y teniendo en cuenta el alcance y la efectividad de las hondas, podría haberse dado el caso de que en el fase final de la batalla los ocupantes de la colina de Andagoste hubieran luchado "espalda contra espalda" para poder defender la posición (Unzueta y Ocharán, 2006).

Finalmente, se ha de señalar las evidencias de dos posibles salidas de la posición, que quedan documentadas gracias a la presencia de sendos rastros de materiales localizados, sobre todo de tachuelas de calzado, las cuales, partiendo del núcleo, y cruzando la zona del cerco, se alejan hacia al este (en dirección al vado que cruza el río Bayas) y al oeste. Si se valoran estas evidencias como un abandono de la posición defensiva habría de considerarse que los defensores se vieron obligados a retirase perdiendo por tanto la posición en la colina de Andagoste (Unzueta y Ocharán, 2006).

\section{Explicación de la batalla a partir de las fuentes literarias}

Si bien para cierta historiografía sería durante las Guerras Cántabras (29-19 a.C.) cuando harían los primeros contingentes militares romanos su aparición en el Cantábrico oriental, fuese con objeto de crear una "cabeza de puente" previa a la sumisión de los territorios todavía controlados por Roma, fuese precisamente como consecuencia inmediata de ésta, los datos facilitados por el yacimiento de Andagoste, un campamento militar de carácter temporal, nos muestran que esta presencia ya se daría en el momento histórico anterior, durante el Segundo Triunvirato, ca. los años 40-30 a.C. (Kavanagh y Quesada, 2007; Morillo, 2009; Morillo y Martín, 2005; Ortiz de Urbina, 2005; Plácido, 2009; Quesada y Kavanagh, 2006; Rodà, 2007). A señalar que Ocharán (2006), seguido por Rodríguez et al. (2012) y Ramos y Jiménez (2008), ofrece el año 38 a.C. aproximadamente para la fecha de este combate, a partir de los hallazgos monetales, es decir, durante el gobierno de $\mathrm{Cn}$. Domicio Calvino (cos. Il 40 a.C.), lo que anteriormente había descartado.

Sea como fuere, los hechos que se produjeron en la colina de Andagoste no están relacionados 
con las Guerras Cántabras que promovió el emperador Augusto (27 a.C.-14 d.C.) ni con las intervenciones previas efectuadas por sus generales, T. Estatilio Tauro (cos suff. 37 a.C., cos. 26 a.C.) en el año 29 a.C., C. Calvisio Sabino (cos. 39 a.C.) en el año 28 a.C. y Sex. Apuleyo (cos. 29 a.C.) en el año 27 a.C., preludios a la gran ofensiva acontecida en el año 26 a.C.

La ausencia en Andagoste de acuñaciones posteriores al año 27 a.C., tan frecuentes en otros contextos arqueológicos, como los típicos denarios legionarios de Marco Antonio (cos. I 44 a.C.) (RRC 544/8-39) (Amela, 2012), parece descartar que los hechos acontecidos en este lugar puedan relacionarse con las Guerras Cántabras y más bien tengan una cronología entre los años 40 y 30 a.C. (Amela, 2006, 2009, 2012, 2013; Fernández, 2002; Gil, 2002; Martínez, 2004; Morillo, 2003; Ocharán, 2002, 2004; Ocharán y Unzueta, 2002, 2005; Unzueta, 2007; Unzueta y Ocharán, 1999, 2006), que ejemplifica muy bien el as hispanolatino de la Colonia Lépida (ACIP $1491=\mathrm{CNH} 18=$ RPC I 261), al contrario de lo que se pensó en un primer momento (Ocharán, 2002).

La operación que llevó finalmente al enfrentamiento de Andagoste ha de buscarse en alguna de las campañas lanzadas por los legati de C. Julio Octaviano (cos. I 43 a.C.), posteriormente conocido con el nombre de Augusto, que gobernador Hispania en su nombre durante la década anterior: C. Norbano Flaco (pr. 43? a.C., cos. 38 a.C.), gobernador en los años 36 y 35 a.C.; L. Marcio Filipo (pr. 44 a.C., cos. suff. 38 a.C.), gobernador en el año 34 a.C.; y Ap. Claudio Pulcher (cos. 38 a.C.), gobernador en el año 33 a.C., todos los cuales recibieron los honores del triunfo ex Hispania, como muestran las Acta Triumphalia (Ocharán y Unzueta, 2002, 2005; Unzueta, 2007; Unzueta y Ocharán, 1999, 2006). Se desconoce el verdadero alcance de estos hechos, que, por repetitivos, no parece que llevaran a un feliz término, aunque la inexistencia de textos acerca de una posible resistencia indígena parece indicar que los romanos llegaron finalmente a alcanzar sus objetivos, aunque sobre estos últimos sólo podemos especular.

Los autores de la excavación descartan que pueda relacionarse este yacimiento con $\mathrm{Cn}$. Domicio Calvino (pr. 56 a.C., cos. 53 y 40 a.C.), gobernador entre los años 39 y 36 a.C., por intervenir este personaje contra los Cerretanos (D.C., 48, 42, 1-3) y contra Bogud de Mauretania (D.C., 45, 48, 1). Pero nosotros no vemos dificultad alguna para que pudiera haber actuado en las actuales tierras alavesas dado su permanencia de tres años en Hispania.

La existencia de un contingente militar en este punto estaría relacionada con la sumisión de las tierras de Vizcaya, Guipúzcoa y la zona montañosa de las provincias de Burgos y Álava. Es quizás el intento de acceder y controlar el paso natural hacia la costa cantábrica en su sector oriental la causa principal y el objetivo que explique la presencia de este destacamento romano en tierras alavesas. Debido a ello, ha de suponerse que las tierras de la Llanada Alavesa y de la zona burgalesa de la ribera del Ebro, en las proximidades de la actual Miranda de Ebro, ya habrían sido asimiladas u ocupadas por Roma (Amela, 2006, 2009; Fernández, 2002; Martínez, 2004; Ocharán y Unzueta, 2002; Ortiz de Urbina, 2005; Unzueta, 2007; Unzueta y Ocharán, 2006).

\section{Reflexiones finales}

La presencia de una fuerza militar romana en Andagoste no ha de extrañar, debido a la presencia de, por su entidad y defensas, significativos oppida cerca de Cuartango, como el de Caranca, al oeste; Arka, al este; o el castro de Enmeneru, en el mismo valle. Sin embargo, más bien sería el intento de acceder y controlar el paso natural hacia la costa cantábrica en su sector oriental la causa principal y el objetivo que explique la presencia de esta fuerza romana en este rincón de Álava. Todo ello puede situarse en el contexto histórico de la guerra de conquista de las tierras montañosas del Cantábrico oriental ubicadas entre cántabros y vascones o como paso previo para el asalto definitivo al solar cántabro, tras la consolidación de este frente oriental en fechas anteriores a las Guerras Cántabras (26 a 19 a.C.) (Ocharán y Unzueta, 2002).

En cualquier caso, todo lo anterior obliga a desestimar la teoría sobre el inicio y desarrollo de las Guerras Cántabras (Unzueta y Ocharán, 2006), que en su momento defendió Syme (1970). Este estudioso consideraba que los territorios del Cantábrico oriental fueron adquiridos por Roma 
como consecuencia inmediata de las campañas efectuadas por M. Mesala Corvino (cos. 31 a.C.) en Aquitania (29-28 a.C.) o como resultado de los ataques procedentes de la ribera del Ebro en el momento previo anterior a la gran ofensiva del año 26 a.C. (Syme, 1970).

Syme (1970) defendía que desde la población de Segisamo (Sasamón, Burgos) partieron tres columnas militares romanas para conquistar el territorio septentrional insumiso a la autoridad de Roma. La columna oriental pudo haber seguido un itinerario no completamente conocido pero del cual sería un testimonio tardío el miliario de El Berrón (Valle de Mena, Burgos) (CIL II $4886=$ HEpOL 10426), mal ubicado en Valmaseda (Vizcaya), estableciendo así pues una relación directa entre una calzada y un frente bélico, que rodearía Cantabria en dirección noroeste en dirección a la ría de Bilbao, lo que implicaría la ocupación de al menos la mitad occidental de la actual provincia de Vizcaya y la del norte de Burgos (Syme, 1970).

El hallazgo de Andagoste muestra claramente que ya en los momentos finales de la República ya se efectuaron operaciones militares en los territorios citados. No se ha de esperar a que la lucha por el poder dentro del seno de la clase dirigente republicana se dirima finalmente en la batalla de Actium (31 a.C.) y en la posterior conquista y anexión de Egipto (30 a.C.), para que Roma se interese por la cornisa cantábrica, sino que su interés es anterior.

\section{Abreviaturas}

ACIP. Villaronga, L. y Benages, J. Ancient Coinage of the Iberian Peninsula. Greek / Punic / Iberian / Roman. Les Monedes de l'Edat Antiga a la Península Ibèrica. Barcelona: Societat Catalana d'Estudis Numismátics. 2011.

CIL II. Hübner, E. Corpus Inscriptionum Latinarum, II. Hispania. Berlín: Berlín-Brandenburg Academy of Sciencies and Humanities. 1869.

CNH. Villaronga, L. Corpvs Nvmmvm Hispaniae ante Avgvsti Aetate. Madrid: J. A. Herrero. 1994.

HEpOL. Hispania Epigraphica. Online database. http://eda-bea.es/

RPC. Burnett, A., Amandry, M. y Ripollès, P. P. Roman Provincial Coinage. Volume I. From the death of Caesar to the death of Vitellius (44 B.C.-A.D. 69). Part I: Introduction and Catalogue. ParísLondon: Bibliothéque nationale de France - British Museum. 1992.

RRC. Crawford, M. H. Roman Republican Coinage. 2 vols. Cambridge: Cambridge University Press. 1974.

\section{Bibliografía}

Amela Valverde, L. (2006). Triunfos en Hispania a finales de la República (36-27 a.C.). Iberia, 9, 49-61.

Amela Valverde, L. (2009). Hispania y el Segundo Triunvirato (44-30 a.C.). Madrid: Signífer Libros.

Amela Valverde, L. (2014). El conjunto monetal de Andagoste. Revista Numismática Hécate, 1, 2540. Recuperado de: http://revista-hecate.org

Fernández Palacios, F. (2002). Lengua e historia del Asón al Cadagua (épocas prerromana y romana). Madrid: Universidad Complutense de Madrid.

Gil Zubillaga, E. (2002). Testimonios arqueológicos en torno al mundo militar romano en Vasconia/ Euskal Herria. En Morillo, A. (coord.), Arqueología militar romana en Hispania (pp. 245-273). Madrid: Polifemo.

Kavanagh de Prado, E. y Quesada Sanz, F. (2007). La arqueología militar romana republicana en España: armas, campamentos y campos de batalla. Panorama de la investigación reciente. En Morillo, A. (coord.), El ejército romano en Hispania. Guía arqueológica (pp, 67-86). León: Universidad de León.

Martínez Salcedo, A. (2004). Erromatairen garaiko zeramika arrunta Euskal Herrian - La cerámica común de época romana del País Vasco. Vitoria/Gasteiz: Gobierno Vasco.

Morillo Cerdán, A. (2003). Los establecimientos militares temporales: conquista y defensa del 
territorio en la Hispania republicana. En Morillo, A., Cadiou, F. y Hourcade, D. (coords.), Defensa y territorio en Hispania de los Escipiones a Augusto (Espacios urbanos y rurales, municipales y provinciales) (pp. 40-80). León: Universidad de León - Casa de Velázquez.

Morillo Cerdán, A. (2009). Los campamentos romanos en Hispania. En Almagro-Gorbea, M. (coord.), Historia militar de España. Prehistoria y Antigüedad (pp. 313-324). Madrid: Real Academia de la Historia - Ediciones del Laberinto - Ministerio de Defensa.

Morillo Cerdán, A. y Martín Hernández, E. (2005). El ejército romano en la Península Ibérica. De la 'arqueología filológica' a la arqueología militar romana. Estudios Humanísticos. Historia, 4, 177-207.

Ocharán Larrondo, J. A. (2002). Monedas perdidas en un combate inédito de las guerras cántabras en el valle de Cuartango (Álava). Monedas partidas, monedas forradas. $X$ Congreso Nacional de Numismática. Actas (pp. 335-341). Madrid: Casa de la Moneda.

Ocharán Larrondo, J. A. (2004). Datación de los depósitos monetales anteriores a Augusto en el País Vasco (Vizcaya, Álava y Guipúzcoa). Kobie, 6, 311-314.

Ocharán Larrondo, J. A. (2006). La Batalla de Andagoste (Cuartango, Álava). Euskonews \& Media, 333. Recuperado de http://www.euskonews.com/0333zbk/gaia33302es.html

Ocharán Larrondo, J. A. y Unzueta Portilla, M. (2002). Andagoste (Cuartango, Álava): un nuevo escenario de las guerras de conquista en el norte de Hispania. En Morillo Cerdán, A. (coord.). Arqueología militar romana en Hispania (pp. 311-215). Madrid, Polifemo.

Ocharán Larrondo, J. A. y Unzueta Portilla, M. (2005). El campo de batalla de Andagoste (Cuartango, Álava). Un precedente de las Guerras Cántabras en el País Vasco. En Fernández Ochoa, C. y García Día, P. (coords.), III Coloquio Internacional de Arqueología en Gijón (pp. 77-80). Oxford: Archaeopress.

Ortiz de Urbina, E. (2005). Autrigones, caristios, várdulos, berones. Contribuciones historiográficas (1983-2003) relativas a su evolución en época prerromana y romana. Vasconia, 34, 47-88.

Plácido, D. (2009). Historia de España. Volumen I. Hispania Antigua. Barcelona: Crítica.

Quesada Sanz, F. y Kavanagh de Prado, E. (2006). Roman Republican Weapons, Camps and Battlefields in Spain: an overview of recent and ongoing research. En Morillo, A. y Aurrecoechea, J. (eds.), The Roman Army in Spain. An Archaeological Guide (pp. 65-84). León: Universidad de León.

Ramos Oliver, F. y Jiménez Moyano, F. (2008). La batalla de Monte Currenchos. Revista de Historia Militar, 103, 207-229.

Rodà, I. (2007). Las guerras cántabras y la reorganización del norte de Hispania: fuentes literarias, epigrafía y arqueología. En Morillo, A. (coord.), El ejército romano en Hispania. Guía arqueológica ( $\mathrm{pp}, 55-66)$. León: Universidad de León.

Rodríguez Morales, J. et al. (2012). Los clavi caligarii o tachuelas de cáliga. Elementos identificadores de las calzadas romanas. Lucentum, 31, 147-164.

Santos, J., Emborujo, A, Ortiz de Urbina, E. (1992). Reconstrucción paleogeográfica de autrigones, caristios y várdulos. Complutum, 2/3, 449-467.

Syme, R. (1970). The Conquest of North West Spain. Legio VII Gemina (pp. 79-107). León: Diputación Provincial.

Unzueta Portilla, M. (2007). Andagoste. En Morillo, A. (coord.), El ejército romano en Hispania. Guía arqueológica (pp, 231-234). León: Universidad de León.

Unzueta Portilla, M. y Ocharán, J. A. (1999). Aproximación a la conquista romana del cantábrico oriental: El campamento y/o campo de batalla de Andagoste (Cuartango, Álava). En Iglesias, J. M. y Muñiz, J. A. (eds.), Regio Cantabrorum (Santander), (pp. 125-142). Santander: Caja Cantabria.

Unzueta Portillo, M. y Ocharán, J. A. (2006). El campo de batalla de Andagoste (Álava). En García-Bellido, M. P. (coord.), Los campamentos romanos en Hispania (27 a.C.-192 d.C.). El abastecimiento de moneda, II (pp. 473-492). Madrid: CSIC - Polifemo. 


\section{Fuentes}

Dión Casio. Historia romana. Libros XXXVI-XLV. Madrid: Gredos. 2004. [Traducción y notas de José $M^{a}$ Candau Morón y $M^{a}$ Luisa Puertas Castaño].

Dión Casio. Historia romana. Libros XLVI-XLIX. Madrid: Gredos. 2011. [Traducción y notas de Juan Pedro Oliver Segura]. 



\section{Panta Rei}

PANTA REI es una revista digital de investigación orientada a la Historia y otras ciencias afines. Su principal objetivo es la transmisión del conocimiento científico, dando una oportunidad también a los jóvenes investigadores que quieren abrirse camino en el estudio de las ciencias humanas y sociales. Se compone de estudios originales relacionados con la disciplina histórica así como su didáctica y difusión. Las diferentes secciones que componen la revista son: artículos de investigación, entrevistas a profesionales, recensiones de monografías de actualidad y crónicas de congresos o eventos científicos relevantes.

Todos los artículos publicados son objeto de un proceso de revisión a cargo de un mínimo de dos evaluadores, que se consideran expertos en el ámbito temático del artículo propuesto. Nuestro deseo es poder ofrecer unos contenidos rigurosos, de calidad y de interés.

EI CEPOAT (Centro de Estudios del Próximo Oriente y la Antigüedad Tardía de la Universidad de Murcia) es la institución encargada de la coordinación y gestión de la revista, desde donde anualmente se lanzará la convocatoria para aquellos que estén interesados en publicar sus trabajos, siempre relacionados con la Historia, Arqueología, Historia del Arte, Didáctica de las Ciencias Sociales, etc.

PANTA REI is a digital journal focused on History and other sciences related to it. Its main objective is the transmission of scientific knowledge by giving also an opportunity to young researchers who want to make their way in the study of human and social sciences. It is composed by original studies related to History, as well as its didactics and promotion. The different sections of this journal are: research articles, interviews to professionals, recensions on monographs about current issues and reports about congresses or relevant scientific events.

All the articles published are subject to a revision process carried out by a minimum of two reviewers who are considered to be experts in the field of the article proposed. Our wish is to offer rigorous contents with quality and being of interest to the reader.

CEPOAT (Centre of Studies of the Middle East and Late Antiquity of the University of Murcia) is the institution in charge of the coordination and management of this journal. This is the centre from where the call for papers will be launched for all the people interested in publishing their papers, always related to History, Archeology, Art History, Didactics of the Social Sciences, etc. 


\section{Normas de Publicación}

El autor se compromete a enviar trabajos originales, que no se encuentren publicados en otras revistas ni en otros idiomas. Así mismo, el mismo artículo no podrá ser presentado en otras revistas mientras dure el proceso de evaluación.

\section{Envío y presentación de originales}

Los artículos se enviarán exclusivamente a través del correo electrónico a la dirección pantarei@um.es. Los textos serán enviados en formato DOC y las imágenes en formato JPEG o TIFF, y con un tamaño mínimo de 2000 px. Éstas no aparecerán incorporadas en el texto, sino enviadas en archivo aparte y correctamente numeradas según su posición en el texto. Junto al trabajo, se rellenará y enviará un documento aparte en el que se especifiquen los datos del autor siguiendo el modelo disponible en la página Web de la revista.

Para la redacción de los trabajos se tendrá en cuenta el Manual de la American Psychological Association, en su sexta edición. La extensión máxima de los trabajos será de 30 páginas. La tipografía será Arial 11, con interlineado sencillo y sin espacio alguno entre párrafos. El texto deberá ir justificado a ambos márgenes y sin sangría en los primeros párrafos. Los márgenes serán de $2,50 \mathrm{~cm}$. En los casos en los que fuera necesario incorporar notas, éstas irán a pie de página, enumeradas consecutivamente, con tipografía Arial 10, interlineado sencillo y justificadas a ambos márgenes.

Una información más detallada se encuentra disponible en la página http://www.um.es/cepoat/ pantarei.

\section{Proceso de valoración y evaluación}

Una vez recibidos los trabajos, la Revista realizará una primera valoración. Si el trabajo enviado se ajusta a las normas de presentación propuestas, la temática es coincidente con la línea editorial de la revista y posee la calidad científica necesaria, será remitido al consejo asesor para una primera evaluación. Si no es así en este primer paso se puede rechazar directamente los documentos que incumplan claramente la línea editorial.

Será el Consejo Asesor quien indique a la revista la originalidad, relevancia, estructura, redacción, aparato bibliográfico, etc. del trabajo enviado y, para ello, se designará a dos revisores expertos externos que evaluarán cada uno de los trabajos, que pueden formar parte (o no) de este Consejo Asesor. La selección de los revisores se ajustará a la temática y características metodológicas del trabajo. El nombre y filiación de los autores serán eliminados del trabajo para su revisión, así como los revisores actuarán de manera anónima y confidencial.

Los revisores deberán rellenar un informe de evaluación que centrará su atención en aspectos tales como características formales, originalidad y novedad de los trabajos, relevancia de las propuestas y los resultados, calidad metodológica y validez científica.

Una vez terminado el proceso se decidirá la aceptación o no de los mismos y su publicación en el número que sea pertinente, así como las modificaciones susceptibles de ser realizadas para su final publicación. Dicha notificación se enviará únicamente por correo electrónico, en un plazo máximo de seis meses. 


\section{Publishing rules}

The author is committed to submit original papers not having been published in other reviews or in other languages. In this way, it is not allowed for the same paper to be presented in other reviews during the evaluation process.

\section{Submission and presentation of originals}

The articles will be exclusively submitted by email to pantarei@um.es. The texts will be submitted in DOC format and the images in JPEG or TIFF format, and with a minimum size of 2000 px. Images will not be integrated in the text but sent in another file and properly numbered according to their position in the text. Attached to the paper, a document will be filled out and sent where the author's data will be specified following the model available on the website.

The sixth edition of the Manual of the American Psychological Association will be taken into account for the writing of the papers. The length of the papers must not exceed 30 pages. Typography will be Arial 11 , with simple line spacing and no space between paragraphs. The text must be justified on both margins without indentation in the first paragraphs. Margins size will be $2.50 \mathrm{~cm}$. Where it could be necessary the incorporation of notes, they will be at the bottom of the page, consecutively numbered with typography Arial 10, simple line spacing and justified on both margins.

More detailed information is available on the website: http://www.um.es/cepoat/pantarei.

\section{Examination and assessment process}

The Journal will submit the papers to a first examination once received. If the paper follows the presentation guidelines, the subject agrees with the editorial line of this journal, and possess the scientific quality required, it will be sent to the advisory council for a first assessment. If not, the documents which clearly fail to complete the editorial line may be rejected straightaway in this first step.

The Advisory Council will indicate the originality, relevance, structure, writing, bibliography, etc. of the text to the journal; for this purpose, two outside experts will be designated to review the papers; these experts can be (or not) part of this Advisory Council. The selection of the experts will adjust to the subject and methodological characteristics of the paper. Name and affiliation of the author will be eliminated from the text for its review, in this way experts will act anonymously and confidentially.

The experts will fill out an assessment report which will focus on aspects such as formal characteristics, originality and novelty of the papers, relevance and results of the proposal, methodological quality and scientific validity.

Once the process is finished, the acceptance or not of the papers and its publication in the corresponding edition will be decided, as well as the modifications that may be done for its final publication. This notification will be sent by email within 6 months maximum. 

